\title{
YOU CAN SHOW THEM THE RISK. YOU CAN TELL THEM IT'S RISKY. SO WHY DON'T THEY BELIEVE YOU.
}

\author{
Ingrid M. Martin, California State University, Long Beach, U.S.A. \\ Michael Kamins, State University of New York at Stony Brook, U.S.A.
}

We have a long tradition of communicating risk to consumers through policy interventions, warnings, PSAs, and other types of strategies. There is a quest to determine the most effective way to communicate risk so that consumers will mitigate those risky behaviors by altering their consumption patterns. From a health marketing and public policy perspective, it is imperative that we understand the underlying processes that drive responses to risk information among those consumers who both consume and consider the consummation of risky products. In this session we present three studies that examine different products (tobacco and dietary supplements) and investigate the impact of different types of risk communications (PSAs, tobacco advertising, and announcements of new research findings) on consumers of these products. We consider a wide variation of consumers who may be at risk inclusive of pre-teens and college students who are at different phases of the consumption process (precontemplative, contemplative, and maintenance of usage patterns). We also use a wide variation of methodological tools (projective techniques and experiments) to test our hypotheses. Both factors contribute to the generalizability of our findings and the practical and theoretical importance of our conclusions.

These studies are designed to go beyond what has already been found in the literature to better understand what motivates consumers as they engage in the consideration process for both pre-teens prior to engaging in the risky behavior (Study 1precontemplative) and college students who are trying out the risky product (Study 2 - contemplative). The final paper (Study 3 - maintenance) investigates the continued risky behaviors for smokers who are faced with various types of warnings. Taken together these three papers delve into the lifecycle of risky behaviors at the precontemplative, contemplative, and maintenance stages when faced with information that is targeted to affect the behaviors.

\section{SUPPLEMENT USAGE STATUS AND RISK INFORMATION}

\author{
Ingrid M. Martin, California State University, Long Beach, U.S.A.
} Sayantani Mukherjee, California State University, Long Beach, U.S.A.

\section{INTRODUCTION}

Dietary supplements are a ubiquitous part of consumers' healthy lifestyle (Wang, Keh, and Bolton 2010). Despite their popularity, there is mixed evidence that supplements improve health. Indeed, it is increasingly common for consumers to encounter risk information about the safety of supplement use. For example, a CNN report cites medical studies suggesting that taking supplements such as Vitamin E could harm users in the long term (Cohen 2009). Moreover, supplement users often discount scientific evidence about harmful side effects, which in turn can make them vulnerable to maladaptive behaviors such as self-medicating and overdosing on supplements (Blendon et al. 2001; Block and Peracchio 2006). Hence, from a health marketing as well as public policy perspective, it is imperative to better understand the underlying processes that might drive responses to risk information among different supplement user segments.

In this research, we examine the impact of dietary supplement usage status on how consumers respond to risk information in the media about a particular supplement brand. For example, we address questions such as: do current users who are currently trying out a specific brand of dietary supplement react differently to risk information from those who have had a long term interaction with the dietary supplement? Likewise do these segments differ from potential users who are considering using the dietary supplement brand? Given past research, which notes that complete adaptation to ownership takes time (Strahilevitz and Loewenstein 1998), we suggest that trial users (vs. current users) with a shorter (vs. longer) ownership history with the brand will evaluate the supplement brand less favorably compared to non users/non potential users (i.e. control group). We also examine the impact of risk information on potential users. Further, building on literature on temporal distance and subjective confidence (Gilovich et al. 1993), we explore the role of temporal distance between initiation of product usage and exposure to risk information as an underlying process driving these effects.

\section{METHODOLOGY}

We examine the impact of usage status on consumers' reactions to risk information about supplement brands through four 